\title{
Practical Distributed Fuzzy Sets
}

\author{
Mary Gracelet $\mathbf{J}^{1}$, Dr.G.Velammal ${ }^{2}$ \\ ${ }^{1,2}$ (Associate Professor, Department of Mathematics, Sri Meenakshi Governement Arts College for Women, \\ Madurai,/India)
}

\begin{abstract}
Membership function of a fuzzy set is the generalization of the characteristic function of a crisp set. Membership functions can be generalized using distributions or generalized functions. Distributed fuzzy sets are defined using distributions. In this paper we introduce the concept of Practical Distributed Fuzzy sets and extend the operations of usual fuzzy sets to practical distributed fuzzy sets
\end{abstract}

Keywords: distributed fuzzy sets, fuzzy sets, generalized function, membership functions, practical distributed fuzzy sets

\section{INTRODUCTION}

The characteristic function of a crisp set is a function from a set to \{0,1\}. In 1965 Lotfi A Zadeh[5] introduced the concept of a fuzzy set. The membership function of a usual fuzzy set is a function from a set to the interval $[0,1]$.This has been generalized in several ways. For example an L-fuzzy set has a membership function where the range is a partially ordered set [2]. Type 2 fuzzy sets, intuitionistic fuzzy sets, non stationary fuzzy sets, hesitant fuzzy sets are some of the other generalizations of fuzzy sets.

In our previous paper[6] we had introduced the concept of distributed fuzzy sets which are defined over intervals in the real line For a distributed fuzzy set membership function can be a 'generalized function' or a distribution. In a fuzzy set the value of the membership function $\mu(\mathrm{x})$ is known at every point $\mathrm{x}$. In contrast, in a distributed fuzzy set, only the average membership value over certain intervals may be known.

As we have proved in [6], if the average membership value over every subinterval is known that will correspond to the usual fuzzy set. But in the real world this rarely happens. Precise data will often be unavailable. So $\mu(\mathrm{x})$ cannot be determined at every point. Only an estimate of the average membership value over a certain interval will be known. Distributed fuzzy sets model this situation very well. If it is too much to hope to determine $\mu(\mathrm{x})$ at every point it is too much to expect to know the distribution $\mathrm{T}$ which defines a particular distributed fuzzy set. In other words the distributed fuzzy set is a theoretical concept which models impreciseness of a certain sort. While constructing mathematical models to represent real life situations involving fuzziness of this type we need the concept of practical distributed fuzzy sets.

\section{PRELIMINARIES}

We briefly recall the definition of distributed fuzzy set.

Definition 2.1: Consider a sequence of functions $\left\{f_{n}\right\}$ in $C_{0}(R)$ with the following properties with respect to the interval $[a, b]$.

$$
\begin{array}{lr}
\text { P1 }: f_{n}(x)=0 & \text { out side }[a-1 / n, b+1 / n] . \\
\text { P2 }: f_{n}(x)=1 & \text { in }[a+1 / n, b-1 / n] . \\
\text { P3 }: 0 \leq f_{n}(x) \leq 1 . &
\end{array}
$$

It is well known that such a sequence exists.

Definition 2.2: Let $\left\{f_{n}\right\}$ be a sequence of functions in $C_{0}^{*}(R)$ satisfying P1, P2, and P3 with respect to the interval $[a, b]$ and let $\mathrm{T}$ be a distribution. $\mathrm{T}$ is said to define a distributed fuzzy set on $[\mathrm{a}, \mathrm{b}]$ if

i) $\mathrm{T}$ is a positive distribution

ii) $\lim _{n \rightarrow \infty} T\left(f_{n}\right)$ exists and is the same for any sequence of functions $\left\{f_{n}\right\}$ in $C_{0}^{\infty}(R)$ satisfying the

iii) $\lim _{n \rightarrow \infty} T\left(f_{n}\right) \leq \mathrm{b}-\mathrm{a}$

$$
\mathrm{P} 1, \mathrm{P} 2 \text {, and } \mathrm{P} 3 \text { with respect to the interval }[\mathrm{a}, \mathrm{b}] \text {. }
$$

Definition 2.3: Suppose $T$ defines a distributed fuzzy set over an interval $[a, b]$. Then it is said to have average membership value $m$ over the interval $[a, b]$ where

$$
m=\frac{1}{b-a} \lim _{n \rightarrow \infty} T\left(f_{n}\right)
$$


The average membership value of $T$ over the interval $[a, b]$ will be denoted by $\operatorname{Avgm}_{\mathrm{T}}([\mathrm{a}, \mathrm{b}])$.

\section{PRACTICAL DISTRIBUTED FUZZY SET}

Definition 3.1: A Practical distributed fuzzy set A on an interval [a, b] is defined by specifying

(i) a partition $a=a_{0}<a_{1}<a_{2}<\ldots \ldots \ldots .<a_{n}=b$ and

(ii) the average membership value $m_{i}$ in each interval $\left[a_{i-1}, a_{i}\right]$ where $\mathrm{m}_{\mathrm{i}}$ lies between 0 and 1 .

The average membership value of $A$ in the interval $\left[a_{i-1}, a_{i}\right]$ is denoted by $a v m_{A}\left(\left[a_{i-1}, a_{i}\right]\right)$

\subsection{Operations on Practical Distributed Fuzzy Sets}

First let us recall how operations are defined on the usual fuzzy sets.

Let $A$ and $B$ be two fuzzy sets defined on an arbitrary set $X$ by their membership functions $A(x)$ and $\mathrm{B}(\mathrm{x})$ respectively. Then the standard Complement, Union and Intersection are defined by

$$
\begin{aligned}
& C(A(x))=1-A(x) \\
& (A \cup B)(x)=\max (A(x), B(x)) \\
& (A \cap B)(x)=\min (A(x), B(x))
\end{aligned}
$$

More generally, complement, union, and intersection of fuzzy sets are defined using functions $\mathrm{C}$, $\mathrm{u}, \mathrm{i}$ satisfying the respective set of axioms given below:

$$
\begin{aligned}
& \text { axiom } \mathrm{C} 1: \mathrm{C}(0)=1 \text { and } \mathrm{C}(1)=0 \\
& \text { axiom } \mathrm{C2}: \text { For all } a, b \in[0,1] \text { if } a \leq b \text { then } C(a) \geq C(b) \\
& \text { axiom } \mathrm{C3}: \mathrm{C} \text { is a continuous function } \\
& \text { axiom } \mathrm{C} 4: \mathrm{C}(\mathrm{C}(\mathrm{a}))=\mathrm{a} \text { for each } a \in[0,1]
\end{aligned}
$$

A fuzzy intersection function(t-norm) i satisfies

$$
\begin{aligned}
& \text { axiom i1 : } \mathrm{i}(\mathrm{a}, 1)=\mathrm{a} \\
& \text { axiom i2 : } \quad b \leq d \text { implies } i(a, b) \leq i(a, d) \\
& \text { axiom i3 : } i(a, b)=i(b, a) \\
& \text { axiom i4 : } i(a, i(b, d))=i(i(a, b), d) \\
& \text { function(t-conorm or s-norm) u satisfies } \\
& \text { axiom } \mathbf{u} \text { 1 : } \mathrm{u}(\mathrm{a}, 0)=\mathrm{a} \\
& \text { axiom } \mathbf{u} 2: \quad b \leq d \text { implies } u(a, b) \leq u(a, d) \\
& \text { axiom } \mathbf{u 3}: u(a, b)=u(b, a) \\
& \text { axiom } \mathbf{u} 4: u(a, u(b, d))=u(u(a, b), d)
\end{aligned}
$$

A fuzzy union function(t-conorm or s-norm) $\mathrm{u}$ satisfies

These concepts are easily extended to the Practical Distributed Fuzzy Sets.

\section{Definition 3.2:}

Suppose $\mathrm{X}$ and $\mathrm{Y}$ are Pdfus on an interval [a,b]. This means corresponding to $\mathrm{X}$ we have a partition of $\quad[\mathrm{a}, \mathrm{b}]=\bigcup_{r=1}^{m} I_{r}$ where $\operatorname{int}\left(I_{r}\right)$ are mutually disjoint and the average membership value on $I_{r}$ is specified as $x_{r}$.

Similarly $\mathrm{Y}$ is defined by writing $[\mathrm{a}, \mathrm{b}]=\bigcup_{s=1}^{n} J_{S}$ where $\operatorname{int}\left(J_{S}\right)$ are mutually disjoint and specifying the average membership value $y_{s}$ on $J_{S}$.

Define $K_{r s}=I_{r} \cap J_{s}$

We will prove that $\bigcup_{r, s=1}^{m, n} K_{r s}$ is a partition of $[\mathrm{a}, \mathrm{b}]$

For any set $S$, let $S^{\circ}$ denote the interior of $S$.

Suppose $\mathrm{K}_{\mathrm{r} \mathrm{s}}^{\mathrm{o}} \cap \mathrm{K}_{\mathrm{r}^{\prime} \mathrm{s}^{\prime}}^{\mathrm{o}} \neq \Phi$

$$
\begin{gathered}
\| \in K_{\text {is }} \cap K_{r s^{\prime}}: \Rightarrow x \in I_{r}^{o} \cap J_{s}^{o} \text { and } x \in I_{r^{\prime}}^{o} \cap J_{s^{\prime}}^{o} \\
\Rightarrow x \in I_{r}^{o} \cap I_{r^{\prime}}^{o} \text { and } x \in J_{s}^{o} \cap J_{s^{\prime}}^{o}
\end{gathered}
$$


Which means $r=r^{\prime} \quad$ and $s=s^{\prime}$.

So distinct $\mathrm{K}_{\mathrm{rs}}$ have mutually disjoint interior.

Also $\cup K_{r s} \subseteq[a, b]$

Suppose $x \in[a, b] \quad \Rightarrow x \in I_{r}$ for some $\mathrm{r}$ and $x \in J_{s}$ for some s.

$\Rightarrow x \in I_{r} \cap J_{s}$ for some $\mathrm{r}$ and $\mathrm{s}$

$\Rightarrow x \in K_{r s}$ for some $\mathrm{r}$ and $\mathrm{s}$

$\Rightarrow x \in \cup K_{r s}$ for some $\mathrm{r}$ and $\mathrm{s}$

$\therefore[a, b] \subseteq \cup K_{r s}$

i.e $\bigcup_{r, s} K_{r s}=[a, b]$

Hence $\bigcup_{r, s} K_{r s}$ is a partition of [a,b].

\subsubsection{Practical Distributed Fuzzy Complement}

Definition3.3 Standard Complement:

The average membership value of the complement of $\mathrm{X}$ is defined by

$$
\operatorname{avm}_{X^{\prime}}\left(I_{r}\right)=1-x_{r}
$$

Definition3.4 General Complement:

More generally the PDFuS complement is defined by

$$
\operatorname{avm}_{X^{\prime}}\left(I_{r}\right)=C\left(x_{r}\right)
$$

where $\mathrm{C}$ is any function satisfying axioms $\mathrm{C} 1, \mathrm{C} 2, \mathrm{C} 3$ and $\mathrm{C} 4$.

\subsubsection{Practical Distributed Fuzzy Intersection}

Definition3.5 Standard Intersection

The average membership value of $X \cap Y$ on $K_{r s}$ is defined by $\min \left(x_{r}, y_{s}\right)$

Definition3.6 General Intersection

More generally the PDFuS Intersection is defined by

$$
\operatorname{avm}_{X \cap Y}\left(K_{r g}\right)=i\left(x_{r}, y_{s}\right)
$$

where $\mathrm{i}$ is any function satisfying axioms $\mathrm{i} 1, \mathrm{i} 2, \mathrm{i} 3, \mathrm{i} 4$.

\subsubsection{Practical Distributed Fuzzy Union}

Definition3.7 Standard Union

The average membership value of $X \cup Y$ on $K_{r s}$ is defined by $\max \left(x_{r}, y_{s}\right)$

\section{Definition3.8 General Union}

More generally the PDFuS union is defined by

where $\mathrm{u}$ is any function satisfying axioms $\mathrm{u} 1$ to $\mathrm{u} 4$

$$
\operatorname{avm}_{X \cup Y}\left(K_{Y s}\right)=u\left(x_{r}, y_{s}\right)
$$

\section{Example 3.1}

Cao and Chen [7] had considered an application of fuzzy sets in the field of meteorology. Klir and Folger [8] have discussed this example in their book. We examine a hypothetical situation similar to these cases.

Consider a particular region which receives rainfall uniformly throughout the year, on average. That means the average yearly rainfall is 12 times the monthly average for any month. Suppose records of the annual rainfall received during every year during the nineteenth century are available. We want to consider those time periods which received "Maximum" rainfall. Let $\mathrm{R}(\mathrm{x})$ be the rainfall received in the year $1800+\mathrm{x}$, as per the record. Suppose we define the membership value as follows:

$$
\mu(x)=\frac{R(x)}{4 M}
$$


Where $\mathrm{M}$ is the maximum amount of rainfall received in a quarter.

Clearly, here $\mu(\mathrm{x})$ is the average membership value during the year $\mathrm{x}$. To illustrate the point suppose $M=20 \mathrm{~cm}$. Say in the year $\mathrm{x}=10$, in the first quarter the rainfall received was $10 \mathrm{~cm}$, in the second quarter 20 $\mathrm{cm}$, in the third quarter $15 \mathrm{~cm}$, and in the fourth quarter it was $10 \mathrm{~cm}$. Then the membership value in each quarter is given by the formula (Rainfall in the quarter)/M. Then if the calculation is done for ever y quarter we see that:

\begin{tabular}{|l|l|l|l|l|}
\hline & First Quarter & Second Quarter & Third Quarter & Fourth Quarter \\
\hline $\begin{array}{l}\text { Membership value } \\
\text { during the quarter }\end{array}$ & 0.5 & 1 & 0.75 & 0.5 \\
\hline
\end{tabular}

However the record shows only the rainfall during the entire year- that is $55 \mathrm{~cm}$. That is quarterwise breakup of rainfall received is not available. Based on this available data we get $\mu(\mathrm{x})=11 / 16$ for the entire year. That is the average of $0.5,1,0.75$ and 0.5 . So clearly the available data can be modeled using the concept of practical distributed fuzzy sets rather than usual fuzzy sets.

\section{CONCLUSION AND FUTURE WORK}

In this paper we have introduced the concept of practical distributed fuzzy sets. Since the operations on the usual fuzzy set are easily extended to PDFuS , these sets are easy to deal with. However, theoretically these PDFuS are different from fuzzy sets. These sets are vaguer than the fuzzy sets. Hence while using these sets in fuzzy logic this should be kept in mind. So it is necessary to device measures to measure the fuzziness of a given Pdfus. In our future work these measures will be introduced.

\section{REFERENCES}

[1] I.M. Gelfand , and G.E. Shilov, "Generalized functions ", Academic press, 1964.

[2] George J.Klir, Bo Yuan, "Fuzzy sets and fuzzy logic: Theory and applications", PHI Learning Private Ltd., New Delhi,2012.

[3] L.Schwartz,"Theorie des distributions", Hermann, Paris, 1966.

[4] H. Weyl,"The ghost of modality", Philosophical essays in memory of Edmund Husserl, Cambridge,MA, pp 278-303.

[5] L.A.Zadeh, "Fuzzy sets", Information and Control 8,1965, pp. 338-353.

[6] G.Velammal and J.Mary Gracelet," Generalizing the concept of membership function of Fussy Sets on the real line using Distribution Theory" ,AIJRSTEM 14-112,page21-25.

[7] Hongxing Cao and Guafan Chen, “ Some applications of fuzzy sets to meterological forecasting” Fuzzy Sets and Systems vol-9, issue 1-3,1983, pp 1-12

[8] J.klir and T.Folger , “ Fuzzy sets ,Uncertainty and information” ,Prentice Hall, Englewood Cliffs, NJ ,1988. 